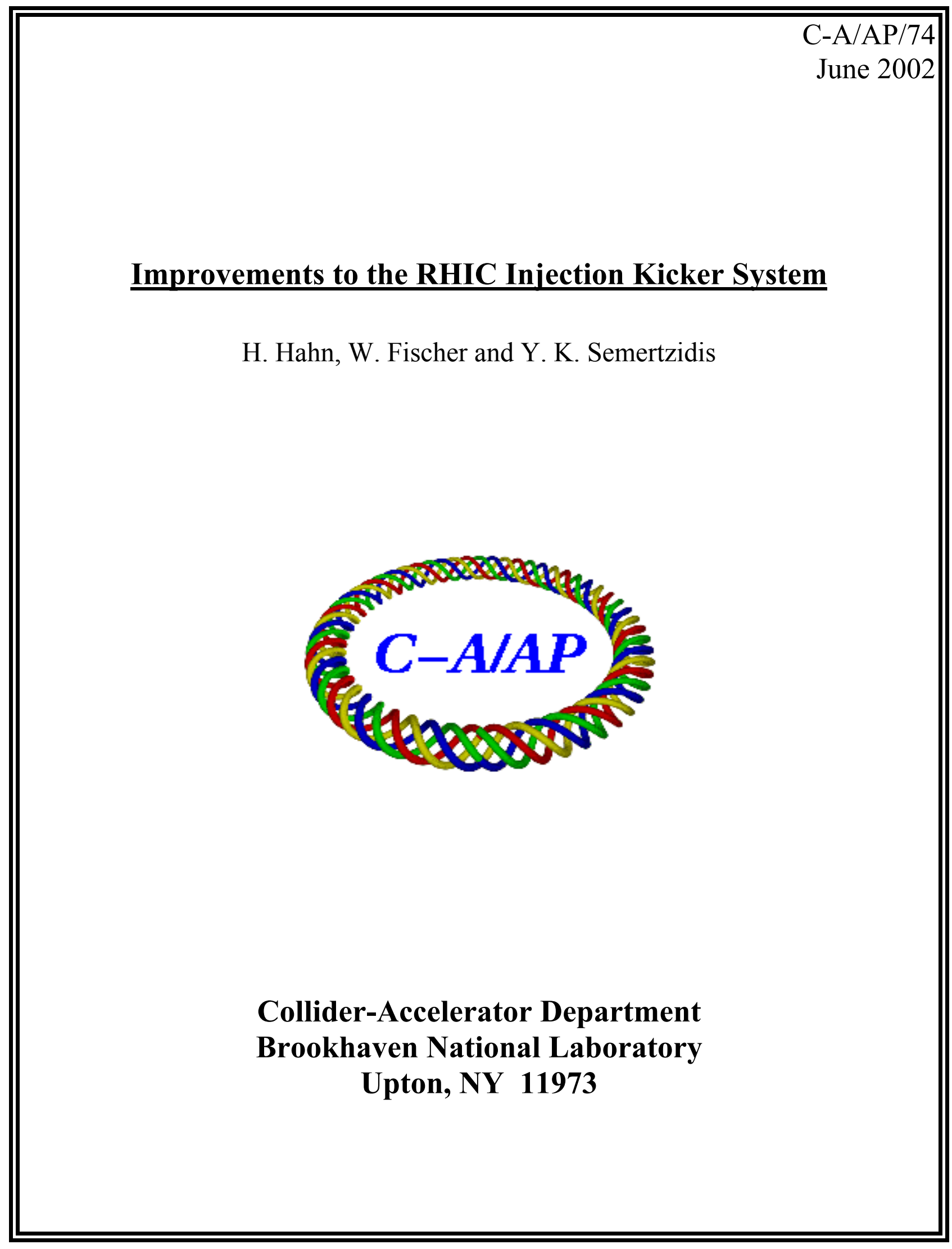




\title{
Improvements to the RHIC Injection Kicker System
}

\author{
H. Hahn, W. Fischer, and Y. K. Semertzidis \\ Brookhaven National Laboratory, Upton NY 11973
}

Executive Summary

The RHIC Design Manual lists under Afuture improvements@ the possibility of filling and operating the rings with a 120 bunch pattern, corresponding to 110 bunches after allowing for the abort gap. However the present performance of the injection kicker systems will require several modifications to achieve this goal. Operation with a 90bunch pattern instead of the standard 60-bunch pattern is already achievable without hardware modifications. In this report changes which promise, but do not guarantee, 110 bunch operation are recommended.

The major components of the RHIC injection system in each ring are the 4 kicker magnets, the thyratrons serving as switches, the thyratron trigger units, the Blumlein pulse forming coaxial tubes, and long coaxial cables between ring and power supply building. Beam measurements during the 2002 run showed a rise time of the injection systems in blue and yellow ring of $110 \mathrm{~ns}$. Injection of 110 bunches was possible although with severe transverse emittance growth. Based on this experience, a system rise time of $95 \mathrm{~ns}$ would seem to be acceptable.

The original concept of the injection system with a transmission line kicker magnet is in principle capable of satisfying these requirements. However voltage breakdown problems forced unorthodox design changes in the kicker magnet. A detailed experimental analysis of each injection system component points to the following hardware modifications, which together promise 110 bunch operation:

- the injection kicker magnets will be terminated with $25 \Omega$ instead of the present $16 \Omega$, promising a $\sim 10 \mathrm{~ns}$ reduction in rise time, however thereby raising the operating voltage from $\sim 31$ to $36 \mathrm{kV}$.

- the home-grown thyratron trigger system consisting of DC power supply and two hard tube trigger units will be replaced by a commercial solid state trigger unit. The new unit eliminates the sensitivity to ac power rectifier fluctuations and reduces the short term pulse jitter to $\sim 3$ ns peak-to-peak.

- the thyratron reservoir ac supply will be replaced by a dc power supply, highly regulated to better than $0.01 \mathrm{~V}$, thereby minimizing drift.

- a fiber optical connection between control room and the thyratron trigger unit will be provided, thereby allowing the operator to adjust slow drifts due to temperature changes affecting the system immediately before injection. 
The above modifications have been implemented in one yellow ring kicker system with typical results documented in Fig.1. Timing jitter over $\sim 7$ min (102 pulses) is $\sim 3 \mathrm{~ns}$ peak-to-peak. Drift in $\sim 10$ hours (nominal storage time) is $5-10 \mathrm{~ns}$ and will be adjusted from the control room. The overall system rise time measurement will have to wait for beam based measurements this fall.

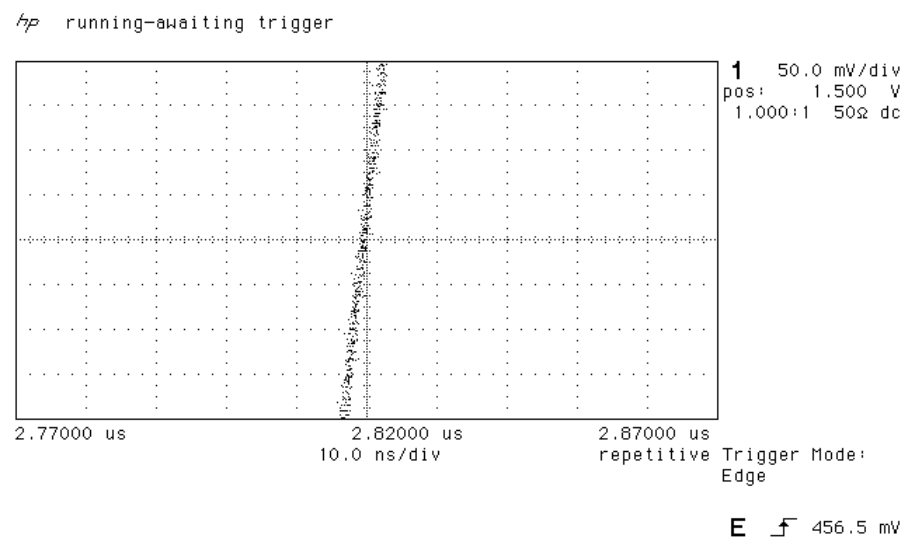

Fig.1A. START TEST: Jitter with 102 pulses $\sim 3 \mathrm{~ns}$ to running-awaiting trigger

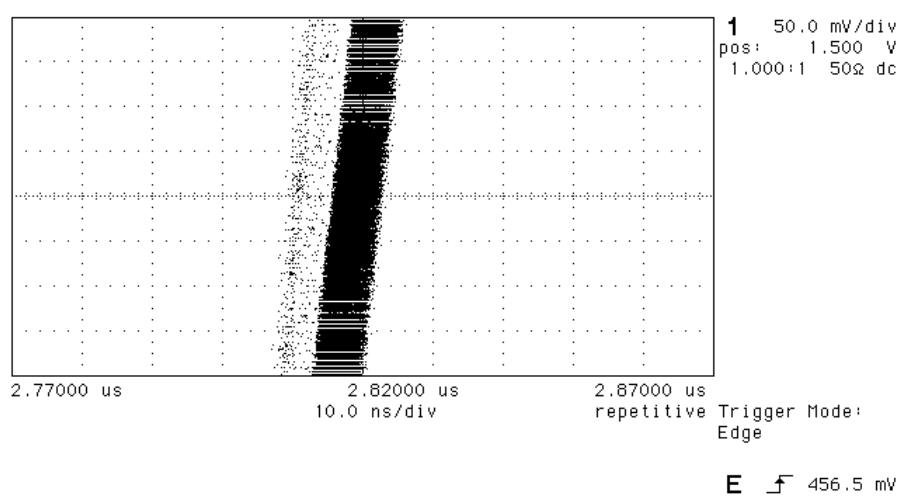

Fig 1B. Jitter \& Drift during $\sim 20$ h, 20,000 pulses, $<8$ ns (note drift \&random triggers $<10 \mathrm{~ns}$ )

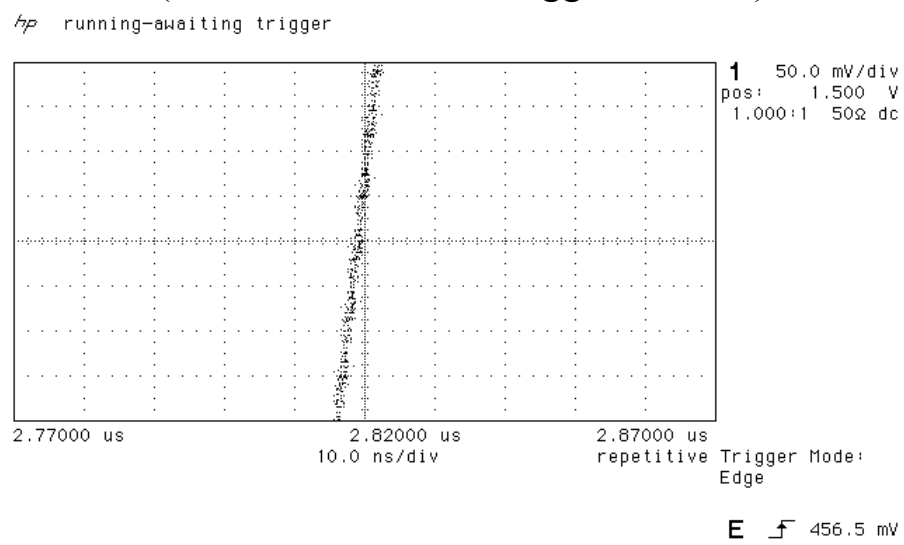

Fig.1C END TEST: Jitter with 102 pulses $\sim 3$ ns 


\section{The all-ferrite kicker magnet}

The RHIC injection kicker was originally conceived as a transmission line magnet with a $25 \Omega$ characteristic impedance, which was achieved by inserting high- permittivity $(\varepsilon>100)$ ceramic blocks between the ferrite blocks.[1] The magnets were successfully used in the 1997 Sextant Test and during the 2001 run. Matched operation of the kicker required $\sim 38 \mathrm{kV}$, a value which was achieved in the laboratory but could not be reliably maintained over million of pulses. Long-term operation without breakdown was improved by mismatching with a $16 \Omega$ termination thereby reducing the operating voltage to $\sim 30.5 \mathrm{kV}$ but at the price of increasing the overall kicker rise time.

With the goal of further increasing the reliability and of reducing the rise time of the kicker system, an all-ferrite kicker magnet was studied and adopted for the 2002 run. [2] In this version, the ceramic blocks were replaced by ferrite, but in order to minimize the conversion cost, the overall geometry and in particular the magnet frame was retained. No magnet failure occurred in this run with the all-ferrite kickers, whereas previously three hybrid magnet needed to be replaced. Results of beam-based rise time measurements are shown in Fig. $2 \& 3$ for the hybrid \& the all-ferrite magnets terminated with $16 \Omega$.

It was not obvious that the converted all-ferrite magnet would retain transmission line characteristics rather than adopt those of a lumped magnet. The effective rise time of a matched transmission line magnet consists of the rise time of the incoming pulse plus the transit time in the kicker. If the magnet is mismatched with a low impedance to gain current and magnetic field at lower voltage, the effective rise time is further increased by the transit time of the reflected pulse. A direct measurement of the magnetic field confirmed the transmission line properties.

The time dependent magnetic field of the all-ferrite kicker was measured using the Faraday effect (1846), which describes the phenomenon that the polarization of a light beam traversing a crystal is changed by an applied magnetic field. This method was successfully developed for the magnetic measurment of a fast non-ferritic kicker for the muon g-2 experiment [3]. In the present setup, a green Ar laser light was used together with an artificially grown TGG crystal which has a large Verdet constant.

The rise time of the magnetic field was measured at two positions in the magnet, $\sim 80$ $\mathrm{cm}$ apart, with one $\sim 20 \mathrm{~cm}$ down-stream from the input and the second $\sim 20 \mathrm{~cm}$ upstream from the terminating resistor. The magnetic field at the magnet input are given in Fig. 4 for $16 \Omega$ and $25 \Omega$ terminations. The magnetic field at the magnet center and magnet output is shown in Fig. 5 for a $25 \Omega$ termination. The transit time to cover the 80 $\mathrm{cm}$ is $\sim 24 \forall 2 \mathrm{~ns}$ in both cases. Since the propagation velocity equals the light velocity times the ratio of characteristic impedance/Z0, one finds a characteristic impedance of $\sim 40 \Omega$.

[1] H. Hahn, N. Tsoupas and J. E. Tuozzolo, Proc. 1997 PAC, Vancouver, BC, p.213

[2] H. Hahn, W. Fischer, V.I. Ptitsyn, J.E. Tuozzolo, Proc. 2001 PAC. Chicago, IL, p. 3705

[3] E. Efstathiadis et al, . submitted to Nucl. Intsr.\&Methds. 

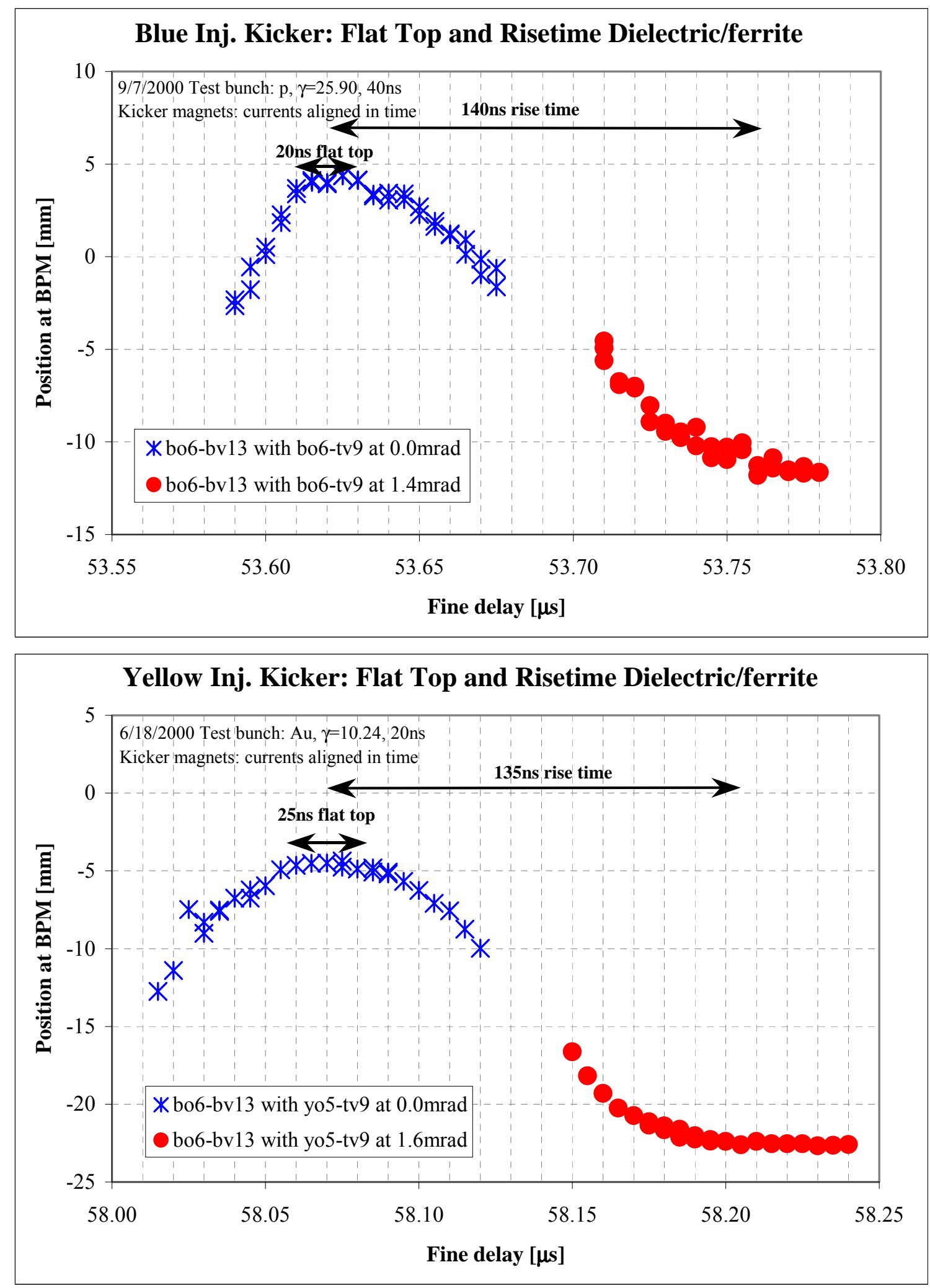

Fig. 2. Beam-based measurement of hybrid kicker magnet with $16 \Omega$ termination 

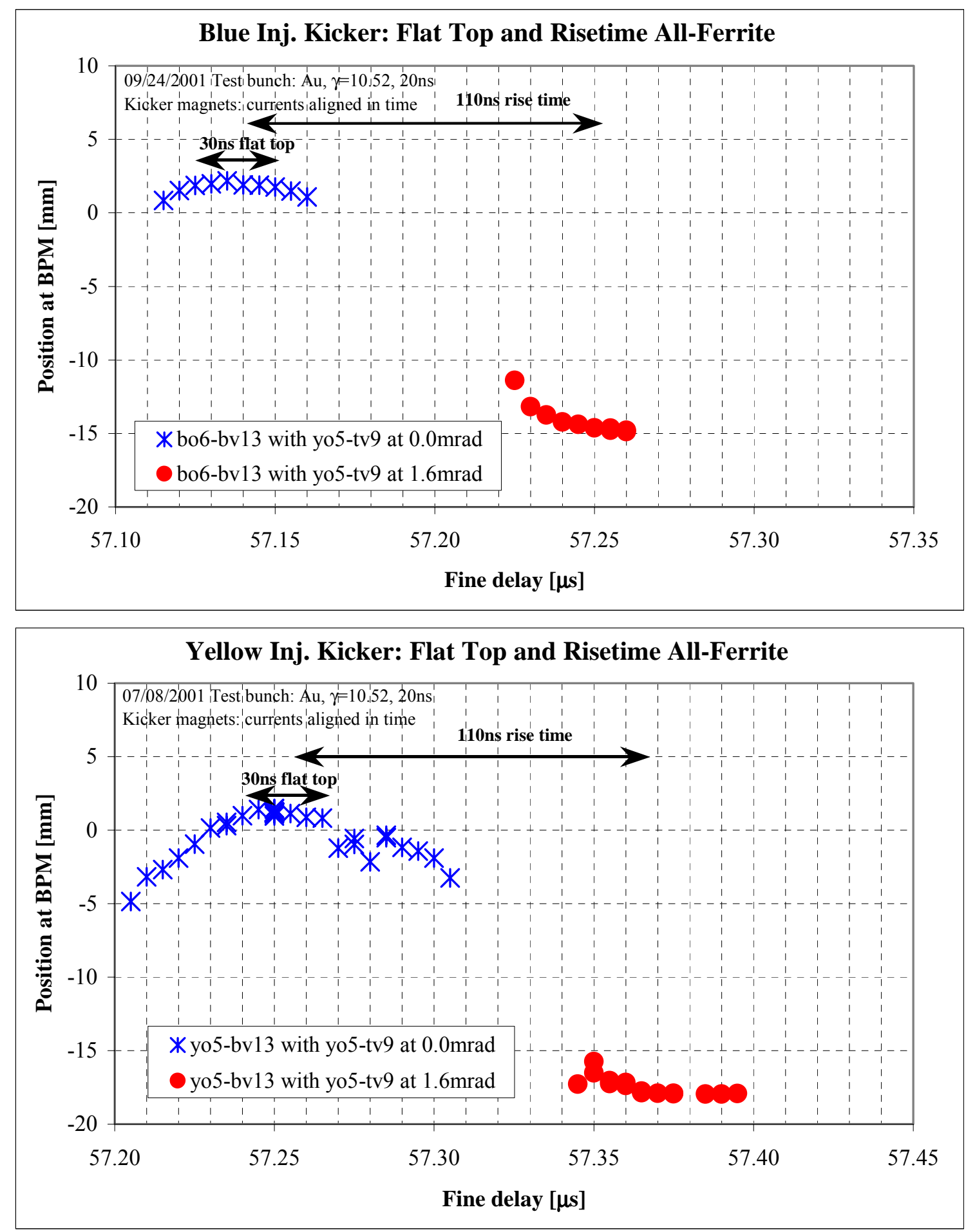

Fig.3. Beam-based Measurements of the all-ferrite kicker magnet with $16 \Omega$ termination 
The full deflecting magnetic field is reached after the current reflected at the terminating resistor has reached the magnet input. The corresponding total current in the kicker magnet is given by

$$
I_{K}=\frac{2}{Z_{K}+Z_{0}}\left(1+\frac{Z_{K}-Z_{L}}{Z_{K}+Z_{L}}\right) U_{0}
$$

where $U_{0}$ represent the Blumlein source voltage and $Z_{0}, Z_{K}, Z_{L}$ is the impedance of the source, of the kicker, and of the terminating load respectively.

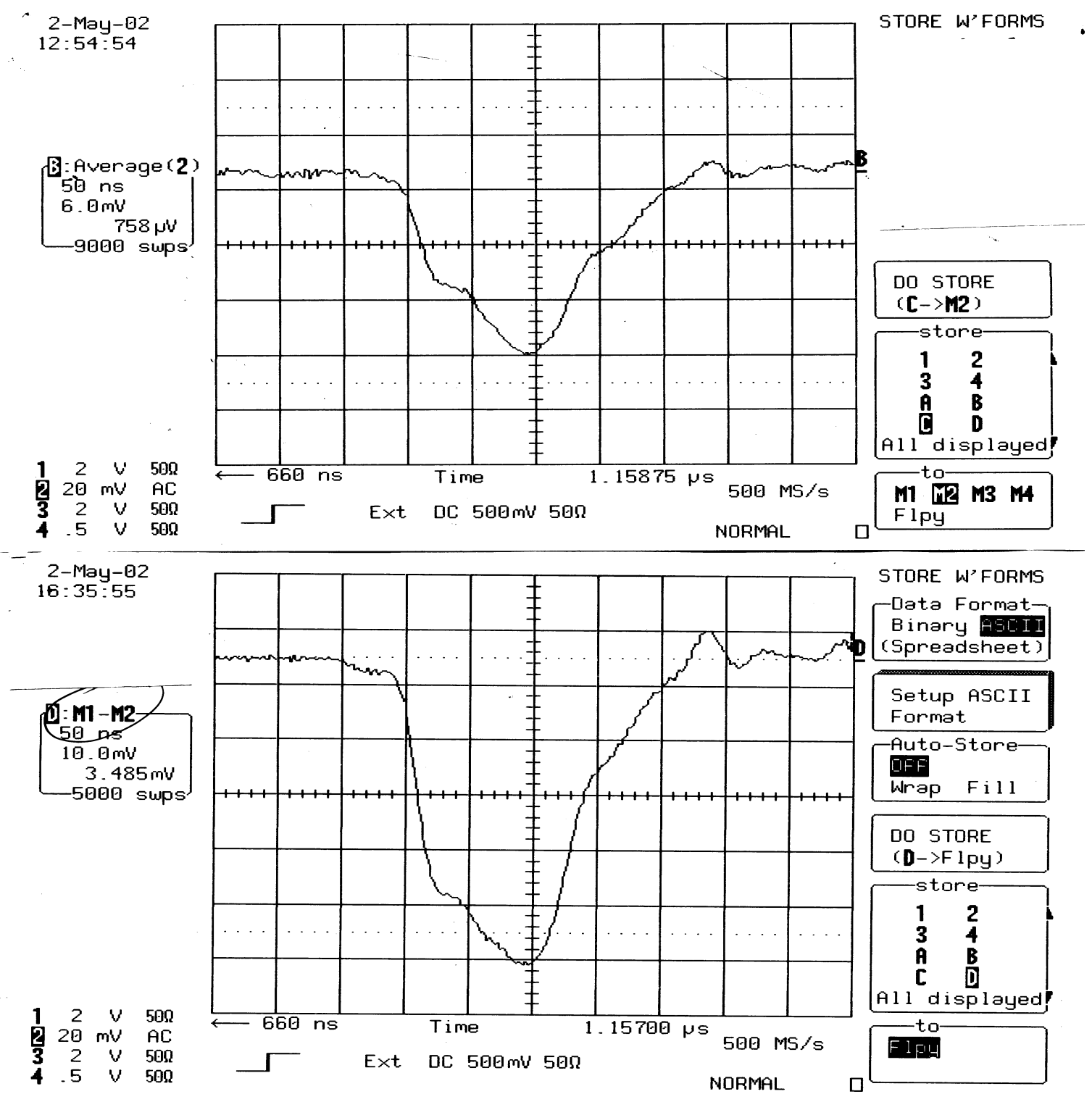

Fig. 4. Magnetic field at $20 \mathrm{~cm}$ downstream from the magnet input (top for $16 \Omega$ and bottom for $25 \Omega$ termination; note scales differ by $\times 1.5$ ) 


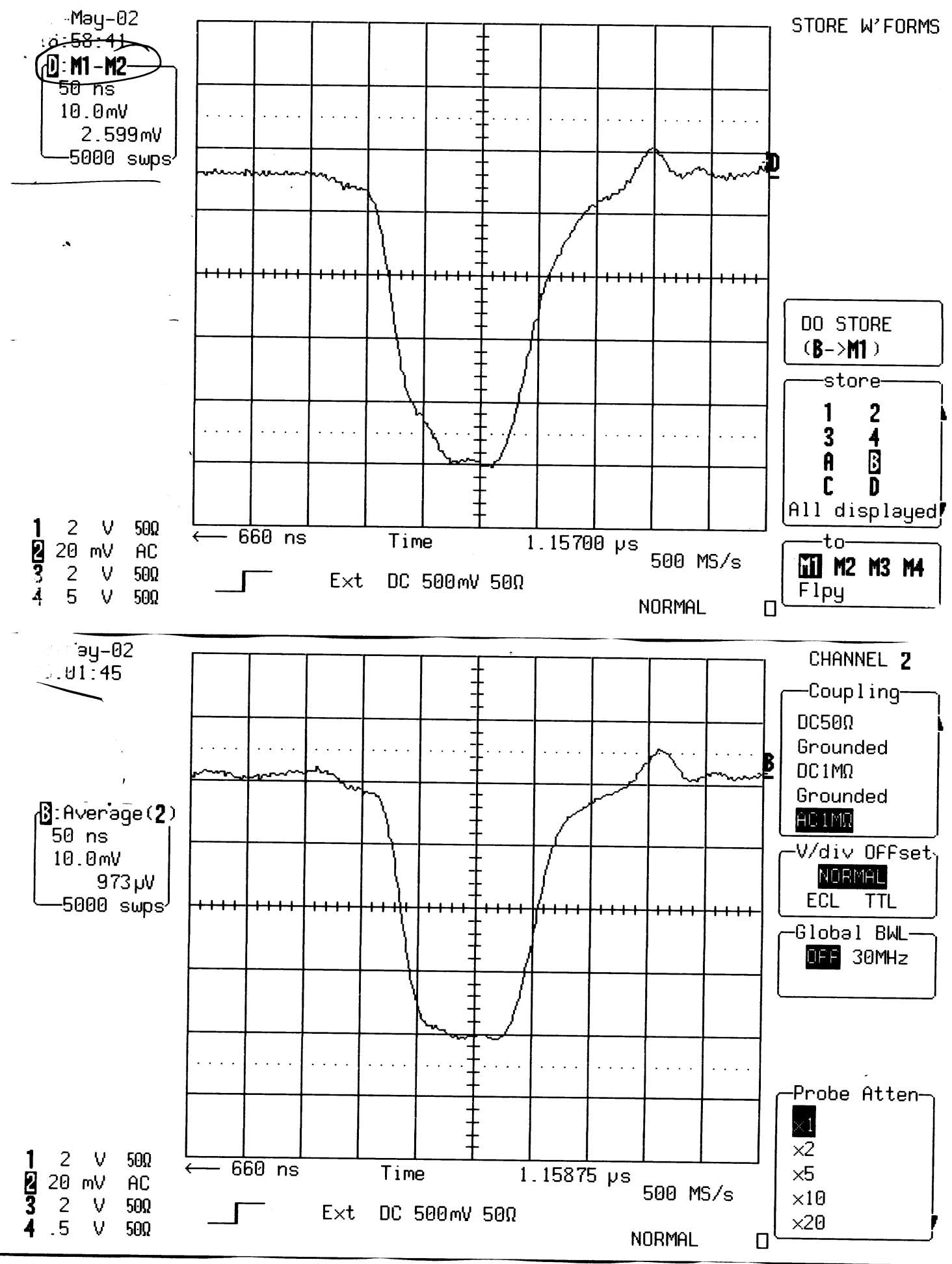

Fig.5 Magnetic field at the middle and at $20 \mathrm{~cm}$ upstream from the magnet output (with $25 \Omega$ termination) 
The total deflecting current is required to be $\sim 1.35 \mathrm{kA}$ at the reduced injection energy of the 2002 run. In the original magnet with $25 \Omega$ characteristic impedance, this corresponded to a $34 \mathrm{kV}$ source voltage. The voltage requirement in the all-ferrite magnet for a matched termination of $40 \Omega$ becomes an excessive $44 \mathrm{kV}$. Mismatched operation with $16 \Omega$ and $25 \Omega$ reduces the voltage to about 31 and $36 \mathrm{kV}$ respectively.

The total injection system rise time was measured at the beginning of the 2002 run to be $110 \mathrm{~ns}$ with the all-ferrite magnet terminated into $16 \Omega$. The rise time is minimized for the matched magnet and can be estimated from the field in Fig. 5 to be $86 \mathrm{~ns}$ $(1.5 \times 24+50)$. Operation with $25 \Omega$ is projected to reduce the rise time by $\sim 10 \mathrm{~ns}$, but verification by beam measurements will be necessary.

\section{Thyratron Trigger Unit}

The switch tube in the Blumlein Pulser is a two-gap deuterium-filled thyratron, EEV $1168 \mathrm{C}(70 \mathrm{kV}, 4 \mathrm{kA})$ ). Proper operation of the thyratron requires a $\sim 2 \mu$ s pre-trigger on grid 1 (G1) and a $0.5 \mu$ s discharge trigger on grid 2 (G2), delay by 0.5 to $3 \mu \mathrm{s}$.. Both triggers, $500 \mathrm{~V}$ on G1 and $1.5 \mathrm{kV}$ on $\mathrm{G} 2$, as well as a negative $150 \mathrm{~V}$ to hold-off the anode voltage, will be provided by a commercial trigger unit EEV MA2709A (a.k.a. "black box"). In the past runs, G2 was triggered with $5 \mathrm{kV}$ to minimize current rise time, however at the expense of exceeding significantly the manufacturer's limit of $2 \mathrm{kV}$. The timing jitter of the thyratron is quoted as $1 \mathrm{~ns}$ typical, with $5 \mathrm{~ns}$ maximum. The trigger timing shift of the commercial trigger unit was tested to be less than 1 ns against line changes of $10 \%($ i.e. $105-120 \mathrm{~V})$.

The thyratron data sheet offers three options for Grid 0 . In the previous runs, $\mathrm{G} 0$ was dc primed with $100 \mathrm{~mA}$. In order to eliminate one power supply (and dependence on its regulation), the G0 grid will be connected to the cathode. The third option, G0 triggered from G1, was explored but showed no measurable improvements,

Each kicker unit in the ring is powered over $100 \mathrm{~m}$ of cable from one Blumlein power supply with its own thyratron. The EEV trigger unit was tested on the thyratron in single

pulse and burst mode, the latter simulating the injection cycle involving the sequence of 4 bunches at a $30 \mathrm{~Hz}$ rate. The anode voltage is applied with a rise time of less then $30 \mathrm{~ms}$, after which the charging is stopped by the "stop" trigger. The discharge trigger must follow in less then $32.3 \mathrm{~ms}$ after the cycle start. It was verified that the discharge timing follows the discharge trigger without being impacted by the charging time, although the current amplitude can be reduced by a short charging time. The current in the kicker terminating resistor is shown in Fig. 6.

The thyratron data sheet gives a nominal rate of rise of anode current of better than $150 \mathrm{~A} / \mathrm{ns}$ but the actual value depends to a large extent on the external circuit. Thus, the rate of rise in the Blumlein pulser is measured to be $\sim 30 \mathrm{~A} / \mathrm{ns}$ at operating conditions, or $45 \mathrm{~ns}$ from 0 to $1.35 \mathrm{kA}$. However, the full rate of anode current rise is only reached after the anode delay time, defined as 0 to $25 \%$, which in the data sheet is given as typically 
$100 \mathrm{~ns}$. In certain power supply configurations, this delay time is minimized by a saturating inductor, but here it was not implemented

The evolution of the current in the terminating resistor after application of the G2 trigger is shown in Fig. 6. From Fig. 6A follows a delay of $\sim 65$ ns until $2 \%$ of peak current is reached and from Fig.6B follows an effective pulse delay of $\sim 85 \mathrm{~ns}$, from $2 \%$ to useable amplitude. Note however, that the signal is transported from the magnet to the power supply room by a $\sim 100 \mathrm{~m}$ cable resulting in an apparently slower rise time. The best measure of the rise time is that of the magnetic field close to the termination shown in Fig.5 B, which gives a similar value.

to running-awaiting trigger

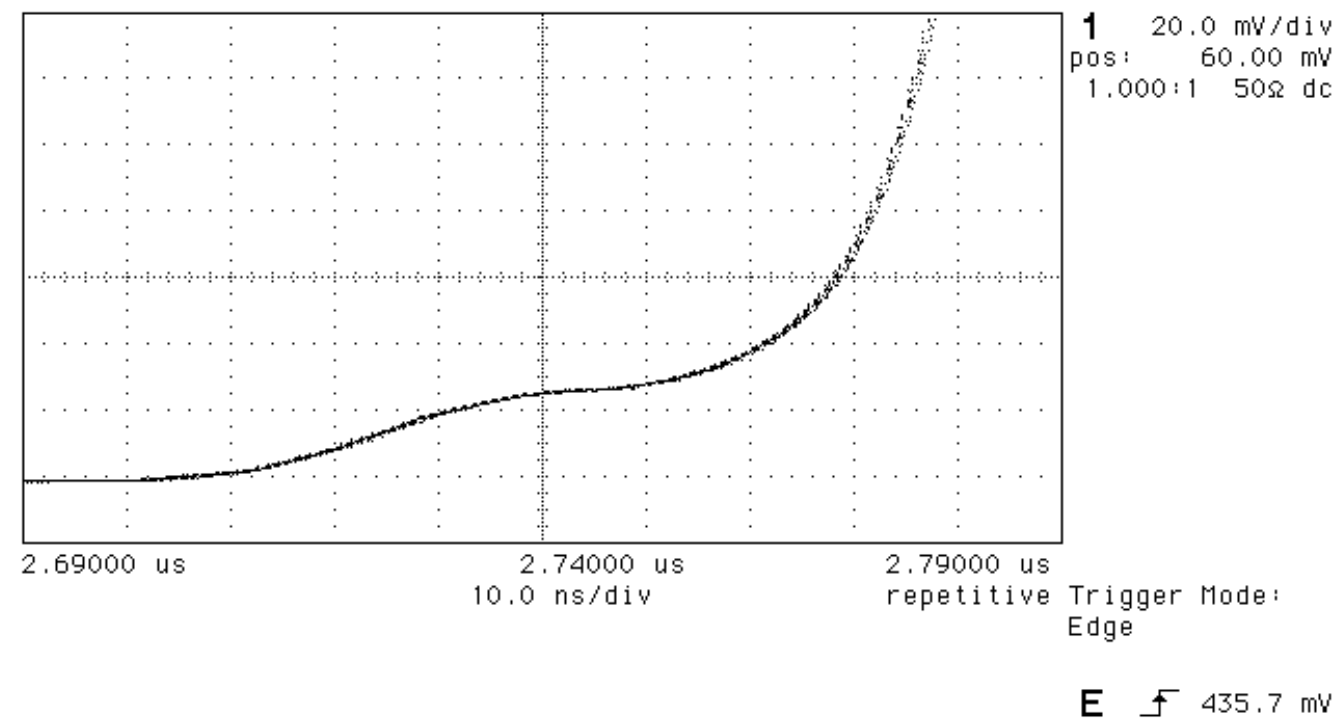

he running-awaiting trigger

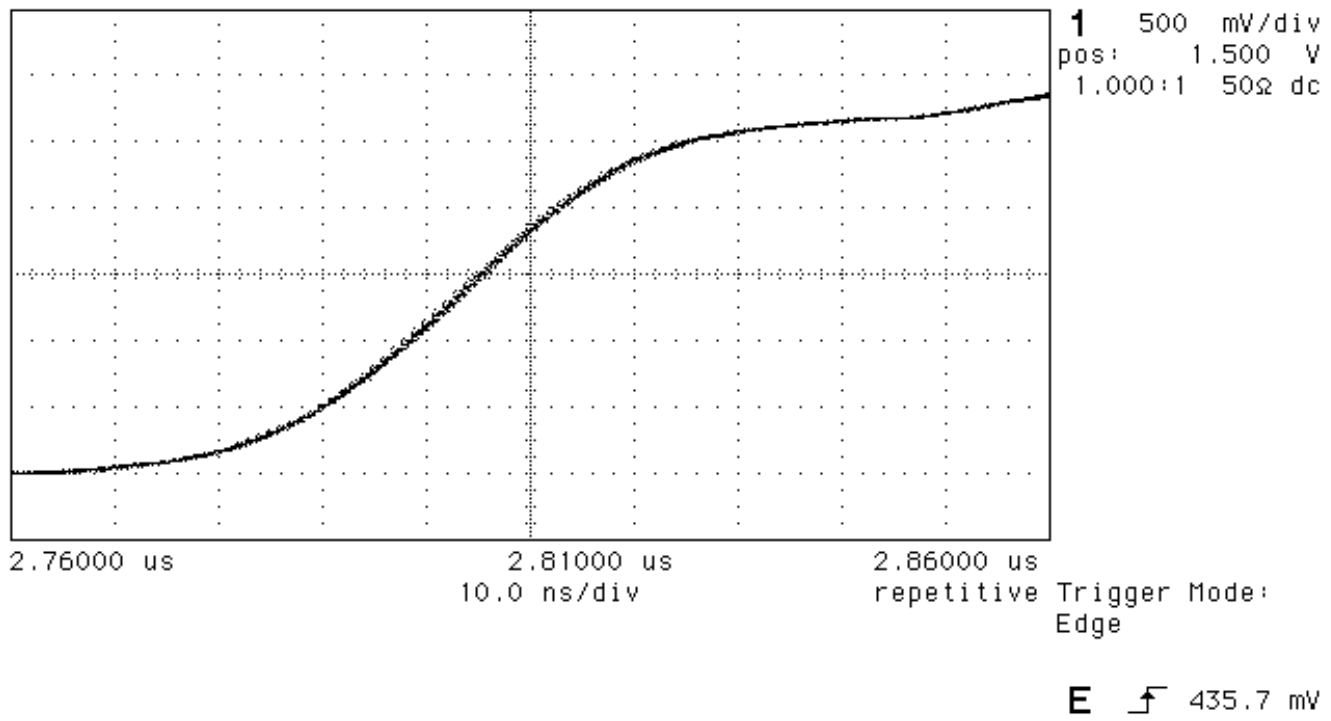

Fig. 6. Kicker termination current delay after G2 trigger at $2.7 \mu \mathrm{s}$.

(Note that the bottom, "B", is shifted by $70 \mathrm{~ns}$ ) 


\section{Reservoir Voltage Power Supply}

The reservoir voltage is the most critical parameter in the quest for trigger stability, both jitter and drift. The dependence of current timing on the dc reservoir voltage is shown in Fig. 7. The data sheet recommends that the reservoir voltage be stabilized to $\pm 0.05 \mathrm{~V}$, which according to our measurements corresponds to typically \pm 3 ns. New DC power supplies, $10 \mathrm{~V} \& 10 \mathrm{~A}$ with $0.01 \mathrm{~V}$ regulation will be used

Achieving the full anode current determines the minimum reservoir voltage.

Operation at the highest level compatible with maintenance of the anode voltage hold-off is recommended. The kicker system runs at a relatively low voltage, well below the 70 $\mathrm{kV}$ peak, so that the reservoir voltage can go to the manufacturer's limit of $6.5 \mathrm{~V}$.

$S_{F}$ running-awaiting trigger

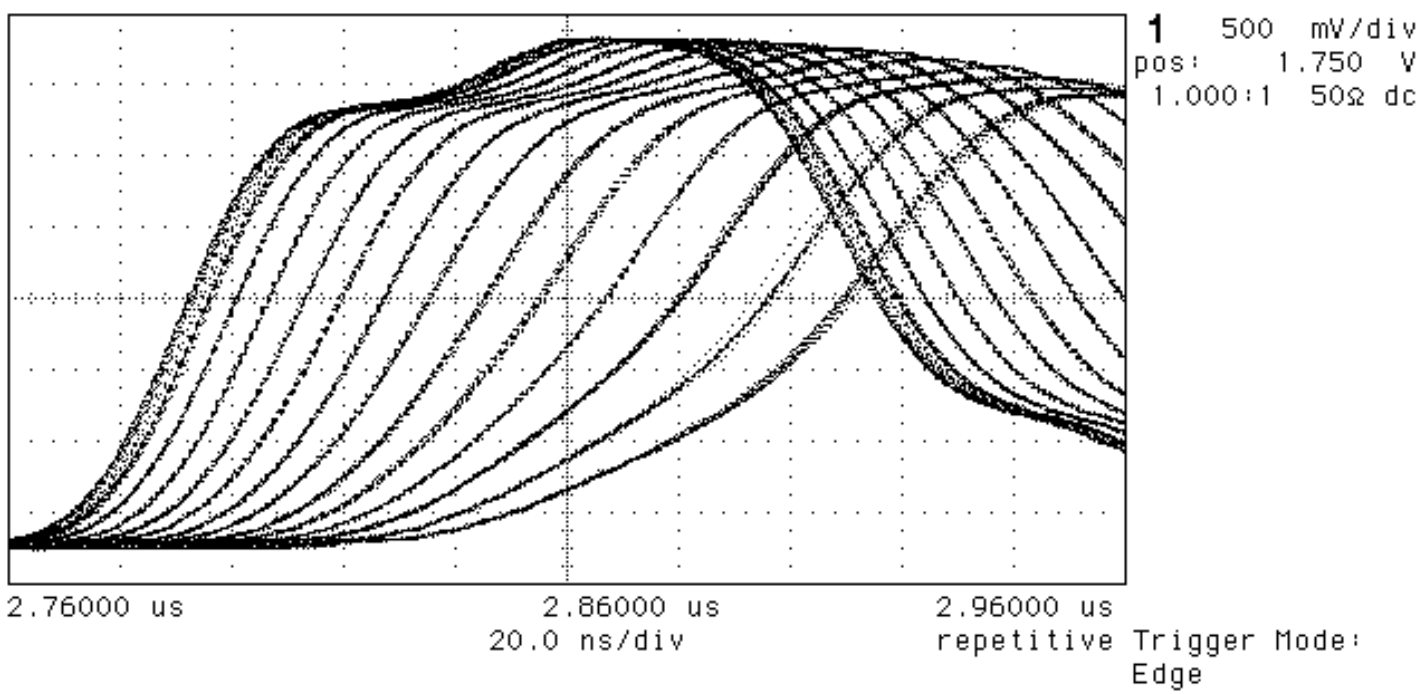

E $f 435.7 \mathrm{~m} /$

Fig. 7. Anode current change with reservoir voltage from 5.0 to $6.3 \mathrm{~V}$ (each line from 10 pulses)

Changing the heater voltage by $10 \%$ has no short term effect on the trigger time, but may contribute to a temperature induced drift. The heater is stabilized by a Solatron transformer.

\section{Acknowledgements}

It is a pleasure to acknowledge informative discussions with Jon Sandberg. Technical Support was provided by Wu Zhang and the members of the Pulsed Power Group, especially Kenneth Hartmann and David Warburton. Last, but not least, the authors would like to thank R. Burns for setting up the magnetic measuring system. 